\title{
The TaSST: Tactile Sleeve for Social Touch
}

\author{
Gijs Huisman* \\ University of Twente \\ Human Media Interaction \\ Group
}

\author{
Aduén Darriba Frederiks ${ }^{\dagger}$ \\ Amsterdam University of \\ Applied Sciences \\ Digital Life Centre
}

\author{
Betsy Van Dijk \\ University of Twente \\ Human Media Interaction \\ Group
}

\author{
Dirk Heylen ${ }^{\S}$ \\ University of Twente \\ Human Media Interaction \\ Group
}

\author{
Ben Kröse $e^{\mathbb{I}}$ \\ Amsterdam University of \\ Applied Sciences \\ Digital Life Centre
}

\begin{abstract}
In this paper we outline the design process of the TaSST (Tactile Sleeve for Social Touch), a touch-sensitive vibrotactile arm sleeve. The TaSST was designed to enable two people to communicate different types of touch over a distance. The touch-sensitive surface of the sleeve consists of a grid of $4 \times 3$ sensor compartments filled with conductive wool. Each compartment controls the vibration intensity of a vibration motor, located in a grid of $4 \times 3$ motors beneath the touch-sensitive layer. An initial evaluation of the TaSST revealed that it was mainly suitable for communicating protracted (e.g. pressing), and simple (e.g. poking) touches.
\end{abstract}

Index Terms: H.5.2 [Information Interfaces and Presentation]: User Interfaces-Haptic I/O, Prototyping

\section{INTRODUCTION}

In our daily interaction with the world around us we not only use our tactile sense for sensory discrimination (e.g. exploring shapes and textures), but also to engage with others around us $[10,26]$. These social touches range from accidentally bumping into a stranger in a busy store, and deliberate handshakes as a means of introduction, to intimate hugs given to loved ones. In human-computer interaction and computer-mediated communication our sense of touch is used in a similar fashion. Haptic feedback allows us, for example, to feel and manipulate virtual objects [33]. Recently researchers have started to explore ways in which haptic feedback can be used to communicate through touch at a distance. Prototypes of these mediated social touch [14] devices include devices to feel someone's presence at a distance [4], devices that augment existing communication channels [5], and devices for intimate contact at a distance [27]. However, most of the current devices only have limited degrees of freedom, restricting interaction to a single touch. In this paper we argue that different types of touch are important in different social situations. To this end, we outline the design process of a device that enables two people to engage in synchronous mediated social touch through a forearm-mounted conductive wool sensor grid, and vibration motor actuator grid. Such a device could serve as an augmentation of existing communication channels, and would prove useful in the study of mediated social touch.

In the next section we will review literature on social touch, mediated social touch, and vibrotactile stimulation of the forearm, that informed the design of the device. Next, we will present the

\footnotetext{
*e-mail: gijs.huisman@utwente.nl

†e-mail: a.darriba.frederiks@hva.nl

¥e-mail: e.m.a.g.vandijk@utwente.n

$\S_{\text {e-mail: d.k.j.heylen@utwente.nl }}$

Ile-mail: b.j.a.krose@hva.nl
}

IEEE World Haptics Conference 2013

14-18 April, Daejeon, Korea

978-1-4799-0088-6/13/\$31.00 @2013 IEEE first prototype of the device, as well as a first user study. The goal of the user study was to see how well different vibrotactile patterns could be perceived as different types of touch. Finally, we present a redesign of the device and provide suggestions for future work.

\section{Related Work}

\subsection{Social touch}

Morrison et al. [26] define three main categories of nonsexual, positively hedonic forms of social touch, namely: simple, protracted, and dynamic. First, "simple" touches involve touches that are brief, intentional and relatively restricted to certain body locations, such as the arm or hand. An example of simple touch is tapping someone on the shoulder to get his/her attention. Second, "protracted" touch involves longer and often mutual skin-to-skin contact where a form of pressure is applied, such as when giving someone a hug. Third, "dynamic" touch involves continuous, often repetitive movement over the skin, as for example in stroking. When these types of touch are applied to the hand, arm, or shoulder in social settings, they can have a number of effects on affiliative behavior and the maintenance of social bonds [26]. Touch can increase the liking of the toucher, and increase the likelihood of compliance to requests $[10,19]$. For instance, when a waitress briefly touches the forearm of a customer (simple touch), the customer is more likely to give a tip [12]. Furthermore, touch can have stress reducing effects [9], for example, holding a loved one's hand (protracted touch) can reduce anxiety caused by an impeding threat [7]. Similarly, stroking (dynamic touch), as compared to other forms of touch, can induce positive affect in infants [30]. Apart from these direct affective consequences, touch can be used to communicate discrete emotions [17, 19]. Studies have shown that people can communicate specific emotions to one another by using simple, protracted, and dynamic touches on the forearm of another person [18]. Participants employed emotion specific touch behaviors to distinguish between different emotions. For instance, simple touches, such as hitting, are associated with anger, whereas dynamic touches, such as stroking, are associated with love [18].

\subsection{Mediated social touch}

The concept of mediated social touch refers to people touching each other at a distance by means of haptic feedback technology [14]. In mediated touch, input devices are used by the toucher to engage actuators that stimulate the tactile sense of the receiver of the touch. For example, Stanley and Kuchenbecker [32] present four actuator designs that aim to mimic human touches, such as tapping (simple), squeezing (protracted), twisting (protracted), and dragging (dynamic). Though these actuators offer promising possibilities for touch at a distance, an issue is that they are relatively large, and thus not suitable to cover a larger surface and still be worn comfortably on the body. More common in prototypes designed for mediated social touch, is the use of vibration motors to simulate a touch. As stated in the introduction, most of these devices have limited degrees of freedom, and are thus 
more geared towards delivering a single, specific tactile sensation, or more symbolic tactile messages, such as vibrotactile patterns representing certain emotional states $[2,8,11,16]$. There are some noticeable exceptions however. ComTouch [5], a hand-held device that was envisioned to augment communication through a mobile phone, uses force-sensitive resistors (FSR) to control the intensity of vibration of vibration motors placed under a single finger. This way, tactile signals can be communicated between two users. ForcePhone [20] incorporates the principles of ComTouch into a more ecologically valid mobile phone platform, and allows users to send pressure sensor controlled vibrotactile messages during a phonecall. Similarly, CheekTouch [29] consists of a 3x3 grid of vibration motors placed on the back of a mobile phone. Touches on the touch screen of one phone, make vibration motors on a second phone vibrate in the same location as where the touch was applied on the touch screen of the first phone. CheekTouch aims to aid with non-verbal and emotional communication during a telephone conversation. These examples allow users to vary the touches they use by changing either intensity (ComTouch, ForcePhone) or location (CheekTouch).

Apart from applications in the design of prototypes, vibrotactile stimulation has been successfully applied in studies investigating similarities between real and mediated social touch $[13,15]$. It is well known from psychological studies that touch can increase the likelihood of compliance to a request $[10,19]$. Similarly, when vibrotactile stimulation was used to simulate a touch to the upper arm, helping behavior increased in a way that was comparable to studies using real touch [15]. Furthermore, the appropriateness of vibrotactile stimulation applied by a stranger to different body sites of the receiver, including the back, abdomen, upper arm, and wrist, is perceived similarly to real touches applied to those body sites [13]. These findings indicate that vibrotactile stimulation is an appropriate method of actuation for mediated social touch. Moreover, the forearm seems an appropriate body-site for mediated social touch to occur $[13,18,19]$.

\subsection{Vibrotactile stimulation of the forearm}

A number of studies have investigated tactile perception of vibratory stimuli (e.g. vibration motors) placed in a grid on the forearm [6, 28, 31]. For example, it has been found that changes in frequency and amplitude are difficult to perceive, while location and duration of, as well as the spacing between stimuli, have a more profound impact on the perception of vibrotactile stimuli $[6,23,28]$. When vibrotactile stimuli are placed in close proximity to body landmarks (e.g. wrist, elbow, edge of the arm) localization accuracy increases [6, 28]. Increasing the inter-stimuli spacing from $25 \mathrm{~mm}$ to $50 \mathrm{~mm}$ increases single-point identification [6]. Decreasing inter-stimuli spacing to $15 \mathrm{~mm}$ creates a more intense sensation and is possibly more appropriate for tactile apparent movement (i.e. a sensation of a single point moving across the arm) $[21,24,28]$. These findings provide valuable insights for the design of forearm mounted tactile displays. For example, spacing between the motors, as well as their location on the arm can be used as design parameters.

\section{THE TASST}

\subsection{Concept}

Based on the considerations outlined above, we designed the TaSST (Tactile Sleeve for Social Touch, Figure 1). The TaSST allows two people to synchronously engage in mediated social touch by touching their own forearm. When two people both wear a TaSST on their arm, a touch to the forearm of the sender is felt as a vibration on the forearm of the receiver. The forearm was chosen because it is relatively sensitive to vibrotactile stimulation [28], easily accessible to touch, and an appropriate location for social touch to occur $[13,18,19]$. The concept of the TaSST is similar
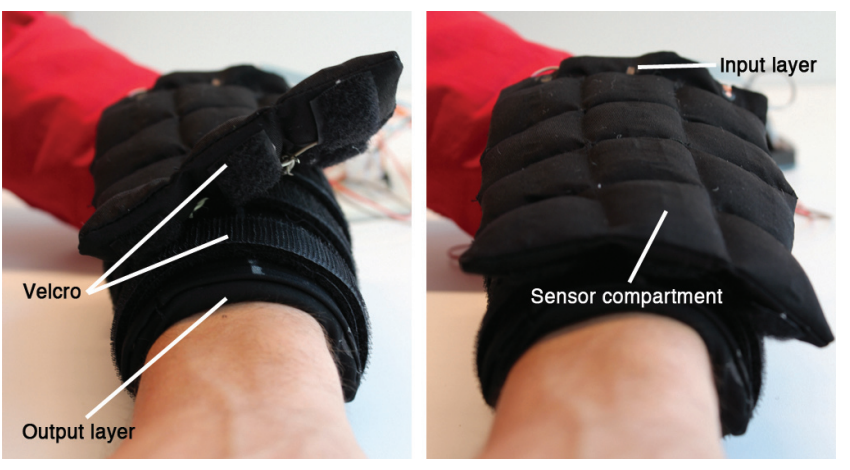

Figure 1: The TaSST. The input layer is attached to the top of the output layer using Velcro.

to ComTouch [5], ForcePhone [20], and CheekTouch [29], but includes both intensity and location as parameters, offering more degrees of freedom for touches to be communicated. Furthermore, the TaSST offers a more direct coupling between the input and output: a touch on the senders own arm will be felt on the arm of the receiver in the same location and with the same intensity.

\subsection{Components of the TaSST}

The system consists of two sleeves that are both composed of an input layer and an output layer controlled by an Arduino Mega microcontroller. The input layer is a 4 by 3 grid of $40 \mathrm{~mm}$ by $40 \mathrm{~mm}$ Lycra pads, filled with conductive wool (Bekeart Bekinox w12/18). When compressed, the resistance of the wool changes, effectively making it a soft, flexible FSR. The advantage of this approach is that the wool-filled compartments are relatively sensitive, detecting changes in force of around $0.4 \mathrm{~N}$. Moreover, the sensors are scalable, do not require a rigid surface to function, and are easily integrated into garments, making the sensor grid suitable to wear on the body.

The output layer is a 4 by 3 grid of pancake style eccentric mass vibration motors (KOTL C1226A001F) attached horizontally to a felt sheet. The motors are $12 \mathrm{~mm}$ in diameter and $2.7 \mathrm{~mm}$ in height. The rotation speed of each motor is controlled by the amount of force that is applied to the wool-filled sensor compartments, so that more force results in stronger vibrations. The vibration motors do not allow for frequency and amplitude to be manipulated independently. However, considering the fact that frequency and amplitude changes are difficult to perceive $[23,28]$, the vibration motors were considered to be well suited for vibrotactile stimulation. We opted for an inter-motor spacing of $40 \mathrm{~mm}$ to allow for relatively accurate single-point identification (e.g. poking) $[6,28]$. Because little is known about the exact spacing requirements for tactile apparent movement without using 'anti-aliasing' methods (i.e. algorithms that create a smooth transition by manipulating the vibration intensity of two motors $[3,22]$ ), we chose a spacing that seems to favor single-point identification over tactile apparent movement.

The output layer is secured around the dorsal side of the forearm using Velcro straps. The input layer attaches to these Velcro straps (Figure 1). The total size of the sleeve is $160 \mathrm{~mm}$ by $120 \mathrm{~mm}$ by $30 \mathrm{~mm}$.

\subsection{Calibration}

The wool in the compartments has the tendency to settle after being touched, producing noise in the data stream. To calibrate the sensors the wool is first fully compressed and then left in idle state to determine the lower and higher threshold. Data from the compartments is smoothed using a low-pass filter and sampled with 


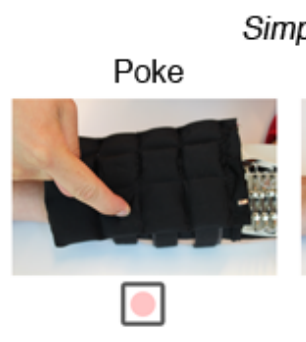

Simple
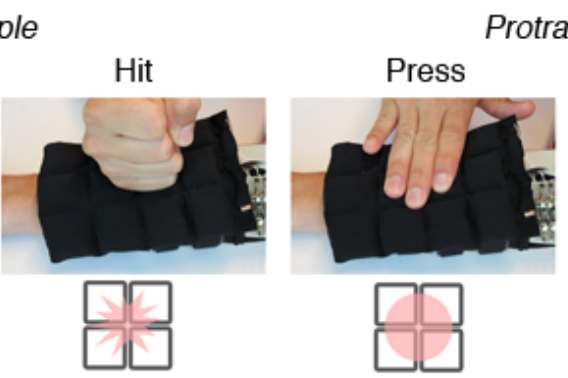

avg. duration $=367 \mathrm{~ms}$

avg. duration $=88 \mathrm{~ms}$

avg. duration $=1223 \mathrm{~ms}$

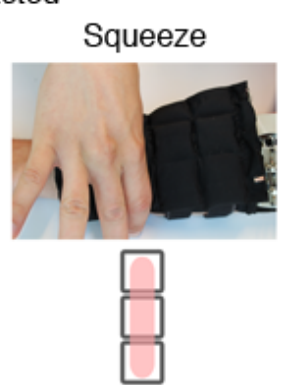

avg. duration $=951 \mathrm{~ms}$

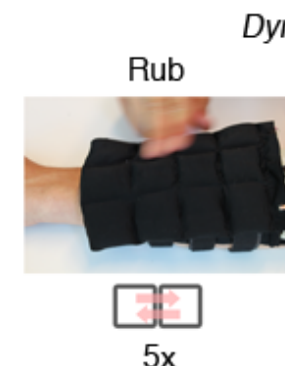

avg. duration $=2846 \mathrm{~ms}$
Dynamic

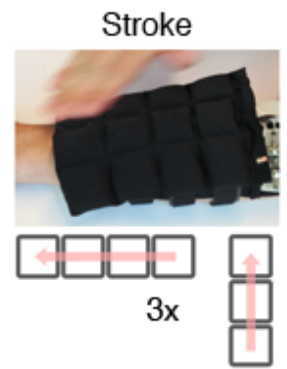

avg. duration $=4666 \mathrm{~ms}$

Figure 2: Prerecorded touches, selected from [18], that were presented to participants. The diagrams indicate how, and how many sensor compartments were touched for each touch.

a 10ms sampling rate. The vibration motors are controlled using PWM (pulse width modulation). Similar to [28] we defined 7 PWM levels resulting in 7 perceptually different vibration levels of the motors. For calculating the PWM values the system uses the conductivity range of the wool between fully compressed (lower threshold) and idle values (higher threshold), dividing this into seven PWM levels.

\section{USER STUDY}

A user study was conducted to assess the capability of the TaSST to communicate simple, protracted and dynamic touches. Our hypothesis was that certain vibrotactile patterns would be perceived as specific touches to the input layer of the TaSST. To this end we recorded simple (poking and hitting), protracted (pressing and squeezing), and dynamic (rubbing and stroking) touches with the input layer of the TaSST (Figure 2). Each touch was applied by the experimenter, and recorded multiple times to arrive at a well recognizable version of the touch (i.e. a prototypical touch of each type). This way, four variations of each touch were recorded, with different locations and orientations (e.g. two strokes over the length and two strokes over the width of the input layer). These locations and orientations were chosen to be close, or further away from bodily landmarks such as the wrist, edge of the arm, and elbow, as well as be oriented along or across the arm. We chose only a small subset of all possible variants in order to have a workable set of stimuli. Because the touches were recorded by the experimenter in a controlled setting prior to the experiment, the recordings should be considered a "best case" scenario. To get an idea of how clearly each prerecorded touch would be recognized when 'played back' through the output layer, we asked participants to imitate the touch they received by touching the input layer.

\subsection{Participants}

The study featured 10 participants ( 8 male, 2 female) who were all students and staff members of a computer science group. The mean age was $28.3(\mathrm{SD}=2.9)$. The average circumference of participants' non-dominant wrist was $169.2 \mathrm{~mm}(\mathrm{SD}=13.1 \mathrm{~mm})$.

\subsection{Procedures}

The participant took place behind an opaque screen, signed an informed consent sheet, and was given a written explanation of the experiment, and the functioning of the TaSST. Then, the measurements of the participant's wrist were taken. The output layer of the TaSST was attached to the participant's non-dominant arm, so that the middle of the output layer was aligned with the centre of the participant's wrist. The input layer was attached to the top of the output layer. Next, the participant was told that someone
Table 1: Crosstabulation of the categories of prerecorded touches (stimulus) and touches coded from the videos (response). Note that one dynamic touch was not coded due to hardware malfunction.

\begin{tabular}{c|cccc|c} 
& \multicolumn{5}{|c|}{ Response } \\
\hline \multirow{4}{*}{ Stimulus } & Simple & Simple & Protracted & Dynamic & Total \\
& Protracted & 22 & 25 & 11 & 80 \\
& Dynamic & 29 & 38 & 20 & 80 \\
\hline \multirow{3}{*}{} & Total & 95 & 87 & 26 & 79 \\
\hline
\end{tabular}

on the other side of the screen would apply a number of touches to an identical sleeve. It was the participant's task to think about how the person on the other side touched his/her sleeve, and to try to imitate this touch by touching his/her own sleeve. In reality the participant received, in random order, the 24 prerecorded touches (Figure 2). After receiving each touch twice consecutively, the participant imitated the touch on his/her own sleeve, and indicated verbally that he/she was ready to receive the next touch. Finally, the participant indicated his/her age, gender, and general comfort level while wearing the sleeve. During the experimental procedure, the participant wore headphones playing white noise to block out the sound produced by the vibration motors. The entire experiment was video recorded from two different angles (top-down as in Figure 2, and from the side).

\subsection{Data analysis}

Based on the description of each touch type by [26] et al. we defined three parameters to assess how well participants were able to imitate the prerecorded touches they received through the output layer of the TaSST. We focussed on how participants touched the sleeve (type of touch), how long they touched the sleeve (duration), and how much of the sleeve they touched (surface area). The type of touch was obtained from annotated video data. The duration of the touches was recorded from the moment of first contact with the surface of the input layer, until the last moment of contact. The data was averaged for the four variants of each touch. Finally, the surface area was obtained by counting all unique sensors that a participant touched while imitating a prerecorded touch. If a participant touched a sensor twice this was counted as a single sensor activation. This way rub, for example, had a surface area of two, since only two unique sensors were activated. Again, the data was averaged for the four variants of each touch. 


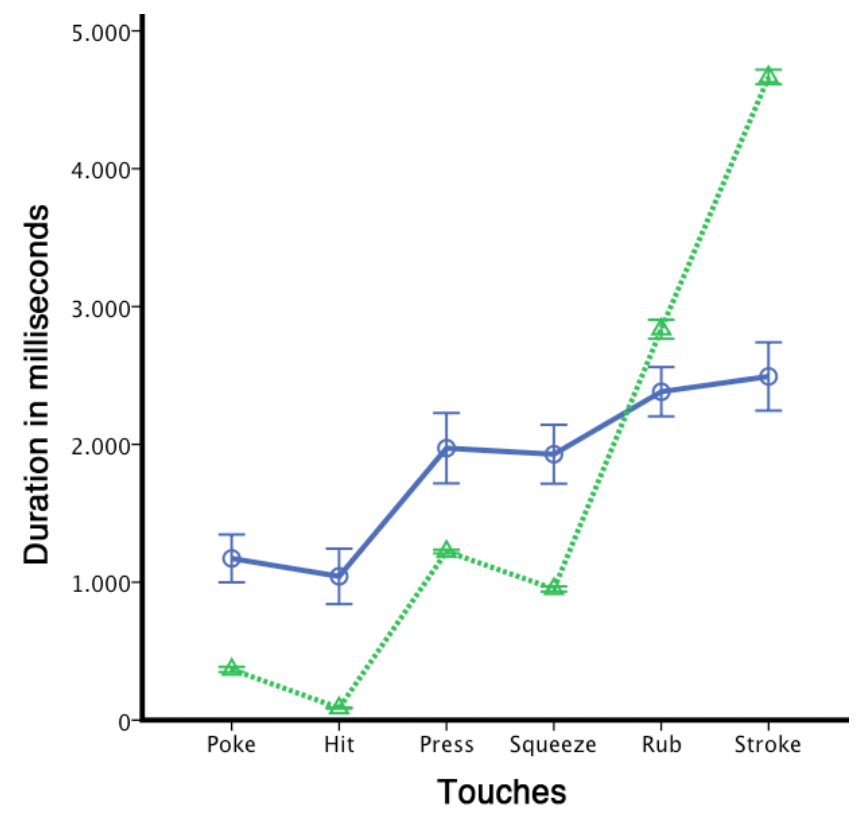

Figure 3: Average duration of prerecorded touches (triangles) and touches by participants (circles). Bars indicate standard error.

\subsection{Results}

\subsubsection{Types of touch}

Based on the videos, two raters, unfamiliar with the order in which the touches were applied, coded each touch made by participants. The coding scheme used was based on [18] and included the following items: rubbing, poking, stroking, massaging, pressing, squeezing, scratching, hitting, tapping, trembling, and pinching. Substantial inter-rater reliability was obtained with Kappa $=.78$, $p=<.001,95 \% \mathrm{CI}(.716, .836)$. In a first discussion round between the raters consensus was obtained on all touches. In a second discussion round, touches were recoded into the three categories of touch (i.e. simple, protracted, and dynamic). From these data, a cross tabulation for the prerecorded (stimulus) and coded (response) touches was made (Table 1). What can be observed from the table is that when presented with simple and protracted touches, participants mostly responded with simple and protracted touches respectively. For dynamic touches however, Table 1 shows considerably more confusion, with responses spread out evenly over all categories. This finding could be explained by the fact that the spacing between the vibration motors $(40 \mathrm{~mm})$ might be less well suited for generating tactile apparent movement [24], making dynamic touches feel more like consecutive pokes, or presses, instead of, for example, a stroking motion. Specific touches that were most used by participants were poking (94), pressing (58), and stroking (39). This indicates that participants used a variety of touches from all three categories, instead of relying on a single form of touch. However, one participant remarked that because the input layer consists of separate thick compartments, he was more inclined to use simple and protracted touches. Moreover, another participant remarked that because the input layer was soft, he had the feeling that he had to apply considerable force for his touch to be registered. Therefore he used more protracted touches. Finally, participants indicated that the sleeve was comfortable to wear $(\mathrm{M}=$ $3.90, \mathrm{SD}=.99$, scale of $1-5$ where 1 is "very uncomfortable", and 5 is "very comfortable"). Wrist size did not influence comfort levels $(F(1,9)=2.97, p>.1)$.
Table 2: Average number of vibration motors activated (stimulus) compared to the average number of sensors activated (response) for all touch types, using a one-sample $t$-test.

\begin{tabular}{llll}
$\begin{array}{l}\text { Touch } \\
\text { type }\end{array}$ & $\begin{array}{l}\text { Average number of } \\
\text { vibration motors } \\
\text { activated (stimulus) }\end{array}$ & $\begin{array}{l}\text { Average number of } \\
\text { sensors activated } \\
\text { (response) }\end{array}$ & Significance \\
& 1 & 2.73 & $p<.005$ \\
\hline Poke & 1 & 2.05 & $p<.000$ \\
Hit & 4 & 3.58 & $n . s$. \\
Press & 4 & 3.63 & $n . s$. \\
Squeeze & 3 & 2.90 & $p<.008$ \\
Rub & 2 & 2.88 & $n . s$. \\
Stroke & 3.5 & &
\end{tabular}

\subsubsection{Duration of touches}

First, the duration of the touches made by participants was compared to the duration of the prerecorded touches, using one-sample $t$-tests. All touches showed a significant difference $(p<.05)$, except for rub $(p<.70)$. This indicates that, except for rub, the duration of the participants' touch differed from the prerecorded touches, as can be observed from Figure 3. For the relatively brief prerecorded touches poke, hit, press, and squeeze (all less than $1300 \mathrm{~ms}$ ) participants overestimated the duration when they were asked to imitate the touch. Conversely, for stroke (about $4600 \mathrm{~ms}$ ), participants underestimated the duration when they were asked to imitate the prerecorded touches. These results were the same when touches were collapsed into their touch categories (i.e. simple touches: poke, hit; protracted touches: press, squeeze; dynamic touches: rub, stroke), and compared to the prerecorded touch categories using one-sample $t$-tests (all touch categories were significantly different from the prerecorded touch categories with $p<.01$ ). However, Figure 3 does show that the data follow a trend that is similar to the prerecorded touches. This trend indicates that, when imitating the touches they received, participants showed a tendency to distinguish temporally between different touches in a similar fashion as the prerecorded touches were distinguished temporally (i.e. duration of simple $<$ protracted $<$ dynamic). Second, touches made by participants were compared to each other, using a repeated measures ANOVA, but no significant differences were revealed. However, a repeated measures ANOVA with Bonferroni correction, for which the touches were collapsed into their touch categories, revealed a significant difference between the duration of simple (1108 ms) and dynamic (2423 ms) touches $(F(1,9)=274,92, p<.05)$. Participants successfully distinguished between these two touch categories on the temporal dimension. This partially supports the trend that can be observed in Figure 3.

\subsubsection{Surface area of touches}

To assess potential differences between the surface area touched by participants in response to a touch they received, a comparison (one-sample $t$-tests) was made between the average number of motors activated for each prerecorded touch and the average number of sensors touched by each participant. These data are shown in Table 2. A significant difference was found between prerecorded touches and touches made by participants for poke, hit, and rub. For poke, and rub participants overestimated the surface area of the touch. A potential explanation for this is that, especially in the case of rub, the surface area was relatively small, but stimulated for a longer duration. It is possible that the fabric sheet, to which the motors were attached, vibrated when motors were activated. In the case of relatively localized touches (i.e. poke and rub), these vibrations might have been perceived as activations of surrounding vibration motors. Conversely, for 
hit, participants underestimated the surface area of the touch they received. This might be due to the fact that hit was very brief in duration (see Figure 3). It is conceivable that the duration was too brief for participants to feel hit as activation of four motors, and instead perceived activation of a smaller surface area (2.05 motors on average). Next, touches were collapsed into their touch categories (i.e. simple touches: poke, hit; protracted touches: press, squeeze; dynamic touches: rub, stroke), and compared to the prerecorded touch categories using one-sample $t$-tests. No significant differences emerged between the average number of motors activated (stimulus) and the average number of sensors touched by participants (response).

\subsubsection{Conclusions}

The results from the user study indicate that participants had the most difficulty in imitating the type of touch when they received a dynamic touch through the output layer. When participants received a simple or protracted touch, they mostly responded with a touch from the same touch category. When the duration of prerecorded touches was compared to the touches made by participants, a similar trend for both types of data was observed. However, all touches, except rub, differed significantly from the prerecorded touches. Still, participants were able to distinguish between simple and protracted touches on the temporal dimension, lending partial support to the trend observed in the duration data. Finally, a comparison between the number of vibration motors activated and the number of sensors touched by a participant, revealed significant differences for the simple touches poke, and hit, and for the dynamic touch rub. No significant differences were found for the protracted touches press, and squeeze. Overall, no significant differences were found between the average number of vibration motors activated and the average number of sensors touched, for the three touch categories.

Overall the data indicate that the first version of the TaSST is more suitable for protracted touches, such as pressing and squeezing. Simple touches were also imitated relatively accurately, but participants had difficulties in estimating the surface area of these touches. Dynamic touches were the most difficult to imitate, which indicates that the current version of the TaSST is not well suited for dynamic touches. In the next section we present a first redesign of the TaSST based on the results from this user study.

\section{REdesign: TASST 1.5}

Based on the results from the user study a number of changes were made to both the input layer and the output layer of the TaSST. Two remarks were made by participants regarding the input layer of the TaSST. A first participant remarked that because the input layer was so soft, he had the feeling he had to press hard for his touch to be registered. A second participant remarked that because the input layer consisted of separate thick compartments, he used more simple and protracted touches. Based on these comments we decided the input layer needed to be thinner, firmer, and smoother. To achieve this, we used a dry felting technique. A $40 \mathrm{~mm} \times 40 \mathrm{~mm} \times$ $10 \mathrm{~mm}$ mold was used to shape the raw conductive wool into square pads with the same dimensions as the mold. This resulted in sensor compartments that were firmer and thinner, but still allowed for 7 distinct levels of force to be detected. The reduced thickness of the sensor compartments also made the input layer smoother overall, compared to the thicker 'cushions' of the original TaSST. These changes should provide users of the TaSST 1.5 with an input surface that is more suitable for dynamic touches, compared to the input surface of the original TaSST. Changes made to the output layer were based on difficulties in the perception of the vibrotactile stimuli, that became apparent in the user study. Here, we considered the overestimation of the duration of received touches, as well as the difficulty in determining the surface area of a received touch.

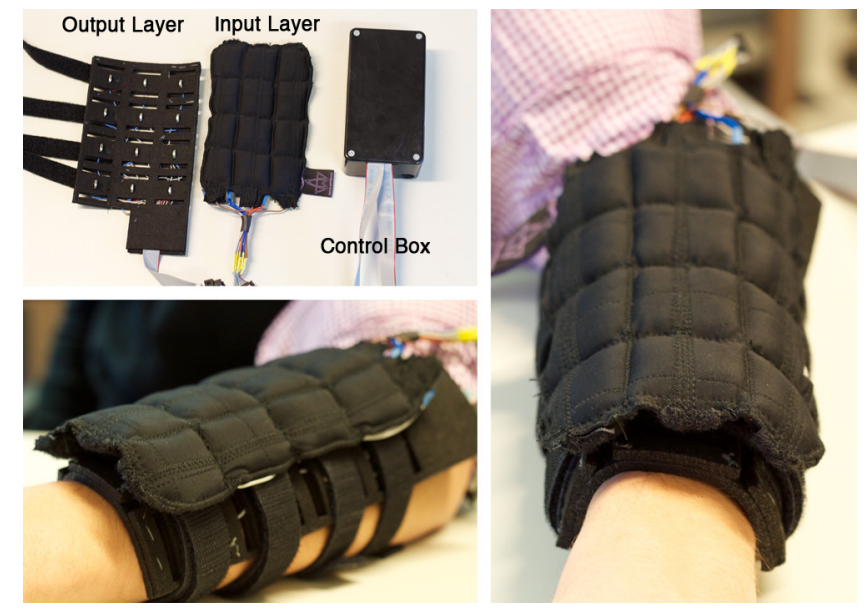

Figure 4: TaSST 1.5. In the top-left the output layer, input layer and control box can be seen.

Regarding the surface area, we suspected that, in some cases, the vibration motors made the fabric sheet, made from firm felt, vibrate. This might explain why participants overestimated the surface area of some of the touches they received. To improve on this issue, we used a laser cutter to remove excess material from the sheet to which the vibration motors were attached. Our aim was to reduce the amount of vibration that could be felt through the entire surface of the output layer of the sleeve. Furthermore, we decided to place the vibration motors perpendicular to the surface of the fabric sheet. Studies suggest that placement of vibration motors in this orientation, improves the perceptibility of vibrotactile stimuli [29, 31]. Intuitively this can be understood by the fact that the direction in which the eccentric mass within the motor casing moves, is towards the skin, rather than moving horizontally over the skin. The changes made to the output layer were aimed at improving the perceptibility of the vibrations generated by the vibration motors. Finally, all of the control hardware necessary to use the TaSST 1.5 was hidden away in a box, which connects to a $\mathrm{PC}$ and power socket. This was purely for practical reasons, making the TaSST 1.5 easier to set up, and less susceptible to hardware failure due to movements made by the user.

\section{Conclusions ANd Future WORK}

In this paper we outlined the design process of the TaSST, a tactile sleeve for synchronous mediated social touch. The design was based on the idea that simple, protracted, and dynamic touches are important in social touch situations. Our goal was to assess to what extent the TaSST could be used to communicate these touch categories at a distance. Results from the user study suggest that the first version of the TaSST was most suitable for communicating protracted touches, and simple touches respectively. Based on these results we made a number of changes to the input- and output layer of the TaSST, in order to make it more suitable for dynamic touches. However, these changes are provisional, and more radical changes to the design of the TaSST might improve its use in the communication of touches from all three touch categories, even further. For example, adding additional vibration motors, and thus reducing the spacing between motors, may make the TaSST more suitable for dynamic touches that benefit from tactile apparent movement [24, 25]. Moreover, algorithms for tactile 'anti aliasing' [24] such as the tactile brush algorithm [22] could be implemented in order to generate believable stroking motions with the TaSST. However, a delay between input and output is expected here. It will have to be investigated what 
kind of impact such a delay would have on the communication of the different touch categories using the TaSST. Finally, vibration motors could be replaced altogether by more responsive voice-coil type actuators. We are currently experimenting with Precision Microdrives' C10-100 Linear Resonant Actuator to replace the vibration motors in the TaSST. On the input side, the size of individual sensor compartments could be reduced. This would create a higher resolution and smoother input surface that might be more appropriate for dynamic touches. Moreover, additional sensors could be added to the input layer. Capacitive sensors for instance, could be used to detect very subtle touches. Combining the data from capacitive sensors with the data from the conductive wool sensor compartments, would make the TaSST sensitive to a wider range of touches. We are currently exploring possibilities for using the wool within the sensor compartments themselves as capacitive sensors.

It has to be noted here that as a first test the TaSST was assessed in controlled conditions in a lab setting. For the first design and redesign of the TaSST it was considered important to study the capability of the TaSST to communicate touches from the different touch categories that are relevant for specific social touch situations. However, the TaSST has not yet been studied in an actual mediated social touch setting. Future research will focus on using the TaSST as a research tool for mediated social touch settings. For example, as an augmentation during telefphone, or video chat conversations [29], mediated communication through a virtual agent [1], or virtual storytelling situations [34]. With this, we hope to study how touches received through the TaSST are perceived, given differing social contexts.

\section{ACKNOWLEDGEMENTS}

This publication was supported by the Dutch national program COMMIT.

\section{REFERENCES}

[1] T. W. Bickmore, R. Fernando, L. Ring, and D. Schulman. Empathic Touch by Relational Agents. IEEE Transactions on Affective Computing, 1(1):60-71, 2010.

[2] L. Bonanni, C. Vaucelle, J. Lieberman, and O. Zuckerman. Taptap: a haptic wearable for asynchronous distributed touch therapy. In Proceedings of CHI'06, pages 580-585. ACM, 2006.

[3] C. Borst and A. Asutay. Bi-level and anti-aliased rendering methods for a low-resolution 2D vibrotactile array. In Proceedings of World Haptics Symposium '05, pages 329 - 335, 2005.

[4] S. Brave and A. Dahley. inTouch: a medium for haptic interpersonal communication. In Proceedings of CHI '97, pages 363-364. ACM, 1997.

[5] A. Chang, S. O'Modhrain, R. Jacob, E. Gunther, and H. Ishii. Comtouch: design of a vibrotactile communication device. In Proceedings of DIS '02, pages 312-320. ACM, 2002.

[6] R. Cholewiak and A. Collins. Vibrotactile localization on the arm: Effects of place, space, and age. Attention, Perception, \& Psychophysics, 65:1058-1077, 2003.

[7] J. A. Coan, H. S. Schaefer, and R. J. Davidson. Lending a Hand: Social Regulation of the Neural Response to Threat. Psychological Science, 17(12):1032-1039, 2006.

[8] K. Dobson, D. Boyd, W. Ju, J. Donath, and H. Ishii. Creating visceral personal and social interactions in mediated spaces. In Proceedings of CHI '01, pages 151-152. ACM, 2001.

[9] T. Field. Touch for socioemotional and physical well-being: A review. Developmental Review, 30(4):367-383, 2010.

[10] A. Gallace and C. Spence. The science of interpersonal touch: an overview. Neuroscience and Biobehavioral Reviews, 34(2):246-59, 2010.

[11] F. Gemperle, C. DiSalvo, J. Forlizzi, and W. Yonkers. The hug: a new form for communication. In Proceedings of DUX '03, pages 1-4. ACM, 2003.

[12] N. Guéguen and C. Jacob. The effect of touch on tipping: an evaluation in a french bar. International Journal of Hospitality Management, 24(2):295 - 299, 2005.
[13] A. Haans, C. de Nood, and W. A. IJsselsteijn. Investigating response similarities between real and mediated social touch: a first test. In Proceedings of CHI '07, pages 2405-2410. ACM, 2007.

[14] A. Haans and W. IJsselsteijn. Mediated social touch: a review of current research and future directions. Virtual Reality, 9(2-3):149-159, 2006.

[15] A. Haans and W. A. IJsselsteijn. The Virtual Midas Touch: Helping Behavior After a Mediated Social Touch. IEEE Transactions on Haptics, 2(3):136-140, 2009.

[16] R. Hansson and T. Skog. The lovebomb: encouraging the communication of emotions in public spaces. In Proceedings of CHI '01, pages 433-434. ACM, 2001.

[17] M. J. Hertenstein, R. Holmes, M. McCullough, and D. Keltner. The communication of emotion via touch. Emotion, 9(4):566-573, 2009.

[18] M. J. Hertenstein, D. Keltner, B. App, B. a. Bulleit, and A. R. Jaskolka. Touch communicates distinct emotions. Emotion, 6(3):528-33, 2006.

[19] M. J. Hertenstein, J. M. Verkamp, A. M. Kerestes, and R. M. Holmes. The communicative functions of touch in humans, nonhuman primates, and rats: A review and synthesis of the empirical research. Genetic, Social, and General Psychology Monographs, 132(1):5-94, 2006.

[20] E. Hoggan, C. Stewart, L. Haverinen, G. Jacucci, and V. Lantz. Pressages: augmenting phone calls with non-verbal messages. In Proceedings of UIST'12, pages 555-562. ACM, 2012.

[21] A. Israr and I. Poupyrev. Control space of apparent haptic motion. In Proceedings of WHC'11, pages 457 -462. IEEE, 2011.

[22] A. Israr and I. Poupyrev. Tactile brush: drawing on skin with a tactile grid display. In Proceedings of CHI '11, pages 2019-2028. ACM, 2011.

[23] L. A. Jones and N. B. Sarter. Tactile displays: Guidance for their design and application. Human Factors: The Journal of the Human Factors and Ergonomics Society, 50(1):90-111, 2008.

[24] J. Kirman. Tactile apparent movement: The effects of interstimulus onset interval and stimulus duration. Attention, Perception, \& Psychophysics, 15:1-6, 1974.

[25] B.-C. Lee, J. Lee, J. Cha, C. Seo, and J. Ryu. Immersive live sports experience with vibrotactile sensation. In M. Costabile and F. Patern, editors, Human-Computer Interaction - INTERACT 2005, volume 3585 of Lecture Notes in Computer Science, pages 1042-1045. Springer Berlin / Heidelberg, 2005.

[26] I. Morrison, L. Löken, and H. Olausson. The skin as a social organ. Experimental Brain Research, 204:305-314, 2010.

[27] F. F. Mueller, F. Vetere, M. R. Gibbs, J. Kjeldskov, S. Pedell, and S. Howard. Hug over a distance. In Proceedings of CHI '05, pages 1673-1676. ACM, 2005.

[28] I. Oakley, Y. Kim, J. Lee, and J. Ryu. Determining the Feasibility of Forearm Mounted Vibrotactile Displays. In Proceedings of HAPTICS'06, pages 27 - 34. IEEE, 2006.

[29] Y.-W. Park, S.-H. Bae, and T.-J. Nam. How do couples use cheektouch over phone calls? In Proceedings of CHI'12, pages 763-766. ACM, 2012.

[30] M. Peláez-Nogueras, T. Field, J. L. Gewirtz, M. Cigales, A. Gonzalez, A. Sanchez, and S. C. Richardson. The effects of systematic stroking versus tickling and poking on infant behavior. Journal of Applied Developmental Psychology, 18(2):169 - 178, 1997.

[31] E. Piateski and L. Jones. Vibrotactile pattern recognition on the arm and torso. In Proceedings of World Haptics Symposium '05, pages 90 -95 . IEEE, 2005.

[32] A. Stanley and K. Kuchenbecker. Design of body-grounded tactile actuators for playback of human physical contact. In Proceedings of WHC'11, pages 563 -568. IEEE, 2011.

[33] R. Stone. Haptic feedback: a brief history from telepresence to virtual reality. In S. Brewster and R. Murray-Smith, editors, Haptic Human-Computer Interaction, volume 2058 of Lecture Notes in Computer Science, pages 1-16. Springer Berlin/Heidelberg, 2001.

[34] R. Wang, F. Quek, D. Tatar, K. S. Teh, and A. Cheok. Keep in touch: channel, expectation and experience. In Proceedings of $\mathrm{CHI}$ '12, pages 139-148. ACM, 2012. 